\title{
International Soft Law: A Review on Paris Agreement
}

\author{
Mulko Asaad Adoa \\ $\mathrm{PhD}$ Candidate \\ Graduate School of Social Sciences \\ Near East University
}

Article History: Received: 11 January 2021; Accepted: 27 February 2021; Published online: 5 April 2021

\begin{abstract}
Paris agreement, despite its many aspects and mixture of hard, soft, and non-obligations that are distinct and contain importance and vitality, however, they are not fully clarified in terms of their boundaries. There are various discussions and debates upon the agreements' success or failure. Additionally, drives behind political parties, further motivation for more compliance with the agreement and to encourage major actors for higher response levels against climate change as it is "the most difficult and complex collective action problem of the world". In this article, challenges to the agreement are noted and possible approaches towards a better future for application and compliance with the agreement are presented. For this purpose, categories of law are looked into in regard to Paris agreement and a number of aspects are highlighted. The interrelation link that exist among these obligations regardless of their forms is explored through the context of Paris agreement and its critical importance is emphasized. Our doctrine approach compares and investigates most recent and relevant studies upon the matter at hand and endeavors to create a better understanding on the importance of Paris agreement.
\end{abstract}

Keywords: Paris Agreement, Soft law, Hard law, Environment, Emissions

\section{Introduction}

The topic of climate change has been a widely recognized matter and this recognition has been increasing. The significance of this issue led to an international agreement regarding climate change in Paris Agreement that was supported by a considerable amount of authorities and various parties. It was stated by the secretary general of the United Nations (Ban Ki-moon) that this covenant is signed with aims and perspectives of the future . It is vivid and clear that the Paris Agreement is in fact a highly successful diplomatic act. The twenty first conference of the parties (COP21) of United Nations with Framework Convention on Climate Change (UNFCC) was called the greatest diplomatic success on a global scale. This is while some have found it to be "weak" and with "false hope" as it is based on a system of 'pledge and review'. This notion holds civil society to carry the burden of nations' compliance with the agreement and their accountability . Regardless, the concept of greenhouse emissions and the effectiveness of Paris Agreement in terms of provisions and regulations is a matter of concern. The Paris Agreement is a mixture of different laws, from hard to soft. It has been said- from lead-up to the agreement and since- the mitigation of emissions are non-binding and obligations regard this matter are 'soft laws' . As it was previously mentioned, the matter of climate change is a global concern and it is highly important to have the parties involved in the agreement to move beyond their obligations in response to climate change. The non-binding obligations were deemed as a precondition for United States and their participation (and ratification), and was also a precondition for major players (e.g. China) to enter the agreement. Flexibility, and ratcheting various obligations in a less time consuming manner was made possible through soft obligations/soft law. However, these notions were faced with challenges in the run towards the agreement. This was felt necessary especially from EU nations as well the countries that are more likely to suffer from climate change (i.e. small islands, and a considerable amount of African countries), which stated that for such agreement to be effective and efficient, it requires hardbindings and hard laws for mitigation and parties' commitments to them .

\section{Paris Agreement in Terms, Bindings and Variables}

The term 'pro-soft law' was a narrative that turned to be dominant factor within the Paris Agreement. This is while the agreement is considered as a legally binding treaty. However, fulfillment of duties and formation of Nationally Determined Contributions (NDCs) are not mandatory and the compliance with the agreement in terms of provisions on non-compliance are stated to be in a 'weak state' . Redistributive justice, facing the governments and in general governance of global climate change that has shifted from 'hard obligations' and is voluntary in regard to commitment and differentiation, are the key aspects that are presented within Paris Agreement. The current shift has significant linkage with neoliberal principles. These principles are known to be the dominant governing matters on a global scales in the past years, and was reflected in Durban and Paris Agreement . The current paper presents strengths and weaknesses of the agreement alongside highlighting challenges and possible approaches. 
Although there are many studies conducted upon hard bindings of obligations, soft law has been less investigated comparatively. This is more specifically regarding treaties and soft law treaties, which the Paris Agreement falls into the same category5. Precision and accuracy of obligations is a key term that correlates, and the compliance to the agreement, particularly the Paris Agreement at hand, seemingly has issues in regard to NDCs. Hard obligations within treaties (e.g. Ozone Agreement, arm control treaties, and WTO agreements) have proven to be more effective, when it comes to states and the intention to change their behaviors. It is partially due to the fact that hard, precise, and clear obligations lead to a higher correlation level between effectiveness and the compliance mechanism that is subsequently followed. Another factor is that treaty-level norms are generally domestic and are under procedures of constitutions, which do not apply nor imply on the instruments and norms of soft bindings.

In the perspective of this research and Paris Agreement, the abovementioned facts regarding soft bindings and the commitment towards them can change in time as compliance with international obligations differ traditionally from soft law perspectives. It was mentioned by Van Asselt that 'signals' proposed within Paris Agreement are important, such as the need and skewness towards changing global economy from being highly dependent on carbon and fossil fuel to a carbon-neutral economy by the year 2050. It is noteworthy that non-state actors (i.e. businesses and institutions) can act as a major role in this shift. Effectiveness in Paris Agreement is defined as holding the global average temperature below $2{ }^{\circ} \mathrm{C}$ above pre-industrial level, and to maintain this limit with more effort into $1.5^{\circ} \mathrm{C}$. Quality and stringency of obligations within a treaty are determinants of the effectiveness level of treaty and its obligations in regard to a specific issue. These are alongside participation level in the agreement as well as the level of compliance with these obligations .

The Paris agreement is broad in terms of participation. However, the quality and stringency of the obligations presented in the agreement and the likelihood of compliance with these obligations can be considered as issues. It is also important to note that the objective and goal of the agreement is well-established and calibrated, having effectiveness merged in terminology of climate change science. Despite the aforementioned facts, under full implementation of the agreement, NDCs (individual countries and their commitment to reduce greenhouse emissions) seemingly have permission over $2^{\circ} \mathrm{C}$

\section{Contextual Setting}

Terms such as 'soft law' is used in this article and it refers to those obligations that are not legally binding within the body of international law. This is contradictory to 'hard law' that are obligations, which are bindings under the implemented international law. Some scholars have made a distinction between these two terms, such as Brunnee and Toope. They note that binding and its extent regarding obligations within the body of international agreements, exhibit a continuum that exists over both hard and soft law. We assume that nations and governments tend to retain this distinction among different obligations, which whether are under international law and the ones that are not. Therefore, in this article, the term 'hard law' refers to those obligations that are under international law and are legally binding, while 'soft law' refers to those obligations that are non-legally binding under international law, and to those that have legal effects, or legal relevance that can be considered significant .

Additionally, legal form and legal characters of a provision is vital to have made a distinction and explained within the contexts. The consent of a state is required within legally binding instruments of treaties . Due to variations in provisions and their legal characters, some have higher levels of authority and compliance and others have lesser comparatively. For legal character, there are a number of elements that can be namely, location (place in which provision takes place), subjects (those addressed by the provision), normative content (requirements, standards and obligations included in the provision), language (tone of being mandatory or recommendatory), precision (contextual qualification and discretions within clauses of provision), and oversight (existence of institutional mechanisms for accountability, compliance and clarifications of the provision). Provisions within a treaty can have variations in terms of legal character. An example would be FCCC that contains an array of provisions (discretionary and contextual language). Commitments of bodies and industrial nations-financial resources and transferring technologies are termed with "as appropriate", "if necessary", and "as far as possible", that clearly provides discretion with having considered timeframes resulting performance in perspective of a specific obligation and its manner, instead of merely performance or non-performance . Regardless, such provisions set a standard framework for compliance/non-compliance, and consequences, regarding a particular provision and are task-driven, which as a result are influential on effectiveness as well as compliance with the provision.

Similarly, Kyoto Protocol, regarding its objectives and timeframe, shows a higher level of standards and settings, combined with a designated compliance system. This is while the protocol contains provisions that vary based on parties' judgement on the term "appropriate" under certain circumstances. This can be of an issue as it is influential on standards regarding compliance/non-compliance. "Demonstrable progress" was required from developed countries participating in Kyoto Protocol by 2005 regarding mitigation commitments. Non-compliance with mitigation was a subject to being enforced through the pre-determined compliance system . However, due to subjectivity, non-compliance may not oblige to such action. Therefore, rigor, although underlying it can be within provisions, binding instruments-legally, can create compulsory means to oblige in terms of practice, which is uncertain. It can be understood that legal characters can differ within a provision with legally binding instruments. When multilateral setting is applies, domestic parties (political) select their obligations based on an equilibrium of 
soft and hard obligations that measures from their capacity and this combination plays a dynamic role for the commitment level to be presented particularly in regard to global environmental issues .

It is important to note FCCC and Kyoto protocol in regard to their provisions and legal character-that differs within the binding instruments-due to their policies for all countries (both developed and developing) in regard to climate change. However, they do not legally bind mitigation commitment for developing countries, which is due to their capacity as it was previously mentioned. Such countries have been proposed to have flexibility in terms of obligations . Although it may seem that developing countries have then no real fear of obligations, a legally binding instrument shows the willingness of political party and clears the extent of international commitment towards a domestic state in a legislative action. A considerable amount of scholars have stated that legally binding instruments are the nexus of credibility for commitments. Through communication of expectations, production of reliance, and generation of compliance, a legally binding instrument can act. These instruments are commonly known to be able to survive changes within political bodies on domestic scale. For instance, India could have used such instruments to enrich its strength regarding climate change regime. However, prior to Paris Agreement, India was reluctant in such negotiations despite its increasing efforts in relation to address climate change and its issues.

\section{Prior Negotiations to the Paris Agreement}

The dynamic of negotiations, which led to the Paris Agreement, surface from Durban Mandate, in which mitigation commitments in regard to climate change was exhibited . Within the mandate of Durban in 2011, parties were to establish and develop protocol (a legal instrument, or consensus outcome) from which the legal force can be applied to all parties involved. The aforementioned terms and their interpretation was based on a treaty which can be binding under the body of international law, and it can be deduced that these applicable protocol could be consisting soft law outcomes. This "agreed outcome with legal force" was included after insistence from India based on their laws and regulations in regard to climate change and climate change regime. Burdens on developing countries regarding mitigation was argued by India that would lead to a new interpretation of UNFCCC, which could be a violation to Common but Differentiated Responsibilities (CBDR) principles. Based on CBDR developed countries are obliged to be leading actors in the realm of emissions and their reduction on a global scale . It was mentioned that a seemingly powerful climate agreement requires formation of legally bindings with having mitigation commitments in the same manner. This was proposed by EU, Small Island States and their alliance , and a number of developed countries and African Group. It is also noteworthy that a number of countries under development have made the same argument in regard to developed countries and that they must be taking such strong binding commitments in comparison with those that are not developed. Additionally, it was stated by the developing countries that their efforts in terms of mitigation has to be under the condition of financial means, and whether or not there is aid and assistance from developed countries. It is commonly believed that legal bindings (hard law) can lead to promotion of appearance for general public view as well as its effectiveness, when judicial enforcement is nonexistent.

Another vital aspect of prior negotiations to the Paris Agreement was the position taken by the United States government. At the time, the republicans were the dominant party in the US senate, which led to the instruments to be designed in a manner that will not need the approval of the senate, and to be under the power of execution from the president. This carried the acceptance based on voluntary means and non-binding commitments of mitigation. After the election of Trump and the intention to withdraw from the Paris Agreement, the aforementioned strategy showed its significance as it allowed a three year gap between the withdrawal and its actual occurrence. This was with the consideration of the fact that the outcome of the election was not anticipated as it was carried out. However, this ineffectiveness of withdrawal in a timely manner was referred to a term called 'Trump proof'. It was argued that with Paris Agreement having legally binding instruments for US and NDC to be obliged to the mitigations could have made more obstacles for the US government in terms of withdrawal . It is important to note that having such hard laws implied to the agreement could have held the US government to take part in the agreement from the beginning. Separate non-binding (soft) NDCs were involved for mitigation and its commitments as a core binding agreement that was in favor of US, Japan, China, and India. This approach is the representation of pro-soft law as a narrative. As it was mentioned earlier in this text, this approach was the dominant in the final outcome of the Paris Agreement through a 'treaty soft law' model that was presented.

Referring to the main objective of the Paris Agreement, which was mentioned earlier (to keep the average global warming between 1.5 and $2{ }^{\circ} \mathrm{C}$ ), it required all parties to maintain this collective goal on a quick-timemanner regarding their emissions of greenhouse gases. This goal was to reduce sources of emissions whether anthropogenic or sinks of greenhouse gases within this century. Through soft law (non-legally bindings) the obligations in regard to mitigation were specifically proposed. Major obligation of NDCs from the Paris Agreement have been noted in the Article 4 paragraphs 2 and 3, which are as below:

Paragraph 2 of the Paris Agreement states that all parties must be prepared, engage in communications, and to keep and retain a successive NDC with clear intentions of what to be obtained. Domestic mitigation measures are to be followed by each party and the objective of actually achieving the contributions that are being followed.

Paragraph 3 of the Paris Agreement notes that a NDC from a party is to represent its development and progress that is to be more than the existing NDC. Additionally, it has to represent and exhibit the highest degrees 
of the ambitions that are intact with the NDC, with reflections of common but differentiated responsibilities that is in line with their capabilities. This is based on the difference that exists between nations and the existing circumstances .

Considering the movements upon environmental effects of human industries and deterioration of animal welfare as well as their habitats, it is highly important to address such issues and to further investigate, create better understanding on underlying causes, and to increase overall awareness of the public. Since 1990s scholars and political parties have paid attention to this matter. However, currently we are in a state that more than 16,000 species of animals and plants being endangered with extinction. Excessive use of plastic and the economic state that is based on materialism and consumption are among the major roles that contribute to this danger. Fossil fuel and the extent of their usage is another key element. It is noteworthy that global industries are reluctant towards environmental activities as it means reduction in their profit making. Therefore, it is crucial that such elements be considered when investigating the issue of climate change. A vivid example of this reluctance is Trump and his intentions to withdraw from the agreement. Considering the fact that the US is one of the main actors in gas emissions, after China. The table below shows amount of emissions for the top 10 countries in this subject.

\begin{tabular}{|l|l|l|}
\hline Country & $\begin{array}{l}2015 \text { total carbon dioxide emissions } \\
\text { from fuel combustion (million } \\
\text { metric tons) }\end{array}$ & $\begin{array}{l}\text { 2015 per capita carbon dioxide } \\
\text { emissions from fuel combustion } \\
\text { (metric tons) }\end{array}$ \\
\hline China & 9040.74 & 6.59 \\
\hline United States & 4997.50 & 15.53 \\
\hline India & 2066.01 & 1.58 \\
\hline Russia & 1468.99 & 10.19 \\
\hline Japan & 1141.58 & 8.99 \\
\hline Germany & 729.77 & 8.93 \\
\hline South Korea & 585.99 & 11.58 \\
\hline Iran & 552.40 & 6.98 \\
\hline Canada & 549.23 & 15.32 \\
\hline Saudi Arabia & 531.46 & 16.85 \\
\hline
\end{tabular}

Table 1. Carbon Dioxide Emissions in 2015

The climate change and its subsequent issues merely cannot be denied or neglected nowadays as scientists and scholars have made their clear contribution to understanding this phenomenon for over two decades. Relatively, table 2 presents the differences that exist between the Paris Agreement of 2015 and UNFCCC held in 1992.

Table 2: Comparison of UNFCCC and the Paris Agreement based on key aspects

\begin{tabular}{|l|l|l|}
\hline & UNFCCC 1992 & The Paris Agreement 2015 \\
\hline Number of parties & 196 & 196 \\
\hline Objective & $\begin{array}{l}\text { Achieving stabilization of } \\
\text { concentrations of greenhouse gases } \\
\text { within earth atmospher below } \\
\text { dangerous level for anthropogenic } \\
\text { interference with the system of } \\
\text { climate and its changes. }\end{array}$ & $\begin{array}{l}\text { Enhancement of the Conventions' } \\
\text { implementation and its objectives } \\
\text { with holding the increase of global } \\
\text { temperature bellow } 2{ }^{\circ} \mathrm{C} \text { and to } \\
\text { follow endeavors to reach the limit of } \\
\text { increase to 1.5 C. Creation of } \\
\text { balance between anthropogenic } \\
\text { emissions by removals, sources and } \\
\text { sinks of greenhouse gas emissions } \\
\text { through the second half of current } \\
\text { century. }\end{array}$ \\
\hline Guiding principles & $\begin{array}{l}\text { Protection of future and current } \\
\text { humans in regard to climate system } \\
\text { based on equity and considering } \\
\text { CBDR. }\end{array}$ & $\begin{array}{l}\text { Following the guides of the } \\
\text { Convention in following } \\
\text { objectives, principles of CBDR. } \\
\text { its }\end{array}$ \\
\hline
\end{tabular}




\begin{tabular}{|c|c|c|}
\hline Obligations of all parties & $\begin{array}{l}\text { Make available, formulate, } \\
\text { implement, publishing to update } \\
\text { national programs to mitigate climate } \\
\text { change, efforts for development and } \\
\text { promotion of its applications. } \\
\text { Additionally, diffusion, and transfer } \\
\text { of technologies for sustainable } \\
\text { management and its development in } \\
\text { terms of anthropogenic emissions and } \\
\text { its removals. Promotion of } \\
\text { cooperation and conservation of sinks } \\
\text { and adaptation to climate change, } \\
\text { while noting social, economic, } \\
\text { environmental policies and initiatives } \\
\text { for research, education and increasing } \\
\text { public awareness. }\end{array}$ & $\begin{array}{l}\text { "Refer to paragraphs } 2 \text { and } 3 \text { of the } \\
\text { Paris Agreement that was mentioned } \\
\text { earlier in this paper". }\end{array}$ \\
\hline $\begin{array}{l}\text { Additional obligations of developed } \\
\text { countries }\end{array}$ & $\begin{array}{l}\text { Undertaking initiatives to lead the } \\
\text { fight against climate change; } \\
\text { cooperation and assistance for } \\
\text { developing countries and particularly } \\
\text { those more prone to danger (terms of } \\
\text { costs, adaptation, facilitation, } \\
\text { financial means, and transferring } \\
\text { technologies that are environmentally } \\
\text { friendly. Applicable within } 6 \text { months } \\
\text { from entering the Convention and its } \\
\text { enforcement. Carried out } \\
\text { periodically; measures and policies in } \\
\text { detail with the aim of reducing } \\
\text { anthropogenic emissions to their } \\
\text { levels in 1990s (CO2 and other } \\
\text { gases). }\end{array}$ & $\begin{array}{l}\text { Continuing the fight against climate } \\
\text { change with economy emission } \\
\text { reduction goals; provision of } \\
\text { financial aids to developing countries } \\
\text { with regard to mitigation and } \\
\text { adaptation. Mobilization of climate } \\
\text { change finance, that is beyond those } \\
\text { efforts prior to this stage. Increase and } \\
\text { enhance within the capacity building } \\
\text { support for developing countries. }\end{array}$ \\
\hline
\end{tabular}

\section{Pledge and Review}

Social, economic, and political obligations are more dominant in the Paris Agreement when compared to legal authority. This is due to the mixture of hard and soft bindings (See Lawrence and Wong, 2017). Despite avoiding the usage of the term "pledge and review", the function of this agreement was to facilitate this term. This model is the international cooperation model after the WWII to address means of commitment and it can be pledge of reputation of a nation. This model is a common approach for international cooperation. Through Paris Agreement, all parties involved have obligations towards the targets of the agreement and they are to report their progress and development every five years. Nations are to supervise and review their targets and its development. Parties will be held accountable by national and global civil society. Scrutinizing performance of other nations is encouraged through the agreement based on inclusivity. High levels of participation in a social phenomenon or vice versa, deviant behavior can be maximized . If others are meeting their commitments, it makes it harder for those nations that are not fulfilling their commitments. This happens on a contradictory basis when others avoid their commitments. With higher rates of participation, behaviors can be normalized.

Naming and shaming occurs when countries do not fulfill their commitments or set targets that cannot be achieved. Informal sanctions are the naming and shaming that affect the reputation through public use. Social disapproval leads to higher degrees of cooperation among individuals. Smoking or voter turnout are among socially unwanted behaviors that have been under the influence of naming and shaming. Although, shaming cannot be said to have completely solved the issue, but its influence has been significant and cannot be neglected. This effect extends to institutions and even to international scale. Signatories to treaties are concerned with their reputations and have fear of retaliation. Means of enforcement and adherence have been reported to be under the effects of reputations (e.g. Geneva Conventions, and Universal Declaration of Human Rights). Additionally, shaming was utilized to motivate nations towards humanitarian interventions and its support . Without legal obligations, the system of pledge and review has proven to be effective with over 180 countries' submission to NDCs. Scrutiny and reviewing has been taken place under civil society, and these pledges have been published on the website of UNFCCC. A considerable amount of non-governmental parties have made assessments on pledges and mitigation for measuring goals defined by the Agreement, and have created ranks from inadequate, to medium, and sufficient, and the highest rank of role model, which the latter was not entitled to any country. This ranking was created by Climate Action Tracker that is a non-governmental body.

The initial review on INDC will begin in 2023 and the nations, which did not meet their targets and commitments regarding reduction of greenhouse gas emissions will face negative reputation. Additionally, the 
pace of implementation of the agreement and NDCs will be assessed within the coal sector (import, export and financial means), fossil fuel and its subsidies, alongside the financial aid through pledge of \$100 Billion USD for developing countries to be assisted in terms of low-carbon development (LCD). Within the Paris Agreement, taking responsibility for climate change has been stated and concepts are made clear in different manners. Role of shareholders, those possessing most wealth, lobbies and their power for countering scientific evidence on climate change, producers and manufacturers of fossil fuels, banks supporting extraction of fossil fuels have been addressed within the agreement. Through these means, informal sanctions can be undertaken. A number of banks have already pledged to stop or significantly reduce their actions and financing coal projects.

\section{Paris Agreement, a Treaty Soft Law}

Within the Paris Agreement, effectiveness and its evaluation has been a complex matter as the agreement consists of a unique form of 'treaty soft law'. This term can be related to treaties, in which the language and terminology does not force obligations to the parties, while through operation of pacta sunt servanda, it performs bindings on parties. This therefore creates lack of precision in presentation of the obligation, which can be used as parties as previously mentioned in changes of behavior due to interpretation that can be circumstantial. Such treaties have not been studied thoroughly within the literature rather than a number of case studies .

However, there are studies, in which soft law has been examined based on its forms that are namely, (a) instruments that have been created to become hard law, (b) instruments for meeting the existing gaps within hard law, and (c) instruments that are to have an impact on both hard and soft law and their development . It is noteworthy that treaty soft law does not fit under these notions. Additionally, it is imperative to note that what influences obligation is the content and the degree of precision of the language of treaties, and not their formation. However, this does not imply that provisions constituted in a treaty will be complied with or not. In the light of what was mentioned, the natural complexity of measuring efficacy within the body of law, and more particularly to this research, soft law can be seen. This is among the challenges of those involved with this matter. Obligations with having the content of NDCs considered in the Paris Agreement, can be an instance of treaty soft law. Relatively, submission of NDCs are binding obligations within the agreement, parties are solely to direct their aims for obtaining the goal of reduction in the gas emissions on a global scale through the presented NDCs. In other words, although the submission of NDCs is a mandatory obligation, based on the lack of words such as "must", commitment to the NDC and reaching its goals is not clearly stated to be achieved .

Similarly, within the Paris Agreement, divers and various numbers and forms of NDCs is allowed, which are in fact different in terms of their precision. Economy-wide emission reduction is an obligation that is non-binding but developed countries are under its adoption. Developing countries are motivated in the same direction in a timely manner. However, due to the softness of the said obligations, either developed or developing countries can claim their compliance with these provisions. This can be achieved through targeting the reduction of greenhouse gas emissions based on production of energy/unit. Facilitation of compliance have been done through soft law and its initiatives, or in the process of shifting into hard law and its measurements and its enhancement. It cannot be neglected that there is a potential in the body of soft law in terms of having an impact on the power of binding parties through implication of pressure on those that are relatively slower in adopting. This is while those states that have been complying, will obtain a better stance for their future negotiations, having higher bargaining power

\section{Conclusion}

Considering the aforementioned notions of this study, it can be seen that the concern is upon the matter of whether or not the Paris Agreement will be able to make a significant progress in terms of achieving its goals on a global scale. The environmental issues, which have arisen in the past decade has become a center of attention not merely by scholars, but by authorities and governments as well as public. This is due to its direness on human overall well-being and the enormous impacts of climate change upon human lives. Compliance with the Paris Agreement, particularly from those developed countries and those with the highest rates of greenhouse gas emissions (See table 1) can lead to a consensus among nations in the direction of moving forward against climate change, especially global warming. This notion is of vital importance as the second half of the current century could be exposed to severe consequences from the shift within the climate and atmosphere of the earth, affecting generations negatively. This calls for further urge and encouragement for major actors to take initiatives and go beyond their casual "business as usual" state. As the Paris Agreement has taken a 'bottom-up' strategy for its façade, binding targets and time schedules have created a facilitation for reaching consensus in Paris. It is appropriate to note the proper leadership undertaken by the French government for obtaining this consensus. As it can be followed through this paper, embeddedness of soft laws within the agreement of Paris have made a significant impact on the consensus being reached. Additionally, all parties and their NDCs have been termed as voluntary, established outside the range of agreement.

For effectiveness to be evaluated, there are complications as it can be seen from what was covered throughout this paper up to this state. Considering the importance of such agreements on a global scale, it seems necessary to further encourage parties and authorities to act upon their commitments and NDCs for obtaining progress in terms of sustainability and lowering greenhouse gas emissions. This notion has driven increasing attention towards itself as the number of aware people on this matter have been growing more rapidly in the past few years. Many initiatives have been conducted in various aspects. For instance, marine life protections and water pollution has been a significant subject for both scholars and the public. A number of marine parks have been closed and actions 
have been taken in terms of lowering pollution of oceans from NGOs to individuals (e.g. scuba diving, tourists, and tourism as an industry). Existence of the aforementioned soft, non-bindingness of commitments of NDCs in the agreement of Paris, not only enabled the agreement to be conducted, but further created opportunities for avoiding obstacles regarding developed and developing countries in terms of quality of their commitments as well as their stringency.

This is while sharing of burdens and its debates still cloud the overall negotiations upon climate change as it has for past decades. However, such complexities can take a new formation after the Paris Agreement. As an instance, framework of NDCs is correlated with the perception of fairness by that party, and is related to the actions undertaken by other nations. In occasions, where a government tends to change its behavior based on their political willingness (which happens often), hard law and hard treaties are used through accurate commitments as well as effective, powerful mechanisms for properly addressing compliance/non-compliance, or disputes and their settlements (e.g. case of Montreal Protocol, CITES and WTO agreement). As mentioned earlier, within the Paris Agreement, consequences of not having met the goals of NDCs has not been described precisely. This affects the commitment level on the set NDCs. This is additional to 'weak' mechanisms that are set for non-compliance. These elements can have a negative yet significant influence on the degree of effectiveness for Paris Agreement.

Considering abovementioned statements, for behavior to be catalyzed when is observed, it is more feasible to establish reputation. In case of pledge and review and its efficacy, degree of ease of understanding and accessing targets of NDCs in terms of emission reduction is vital. The fact that China and India alongside some other developing countries stood against review of third-parties is a concern. This was especially against independent institutions (in same structure of International Atomic Energy Agency "IAEA"). This has yielded in a vague measure for transparency within the language and terms of the agreement (Article 13). Vitality of this notion is that the 2014 bilateral US and China agreement is a support for the extent of Paris Agreement. This is associated with looking forward in having India to act more upon climate change compared to its movement up to the agreement, which has been somewhat reluctant.

Regardless of the extensive amount of countries that have been involved in the Paris Agreement (196 countries), it is noteworthy that three major greenhouse gas emissions are the United States, China and India (over 50\% of total carbon emission on the globe-refer to table 1). This percentage can be increased in the future if proper means have not been implemented, or these nations are not enforced to lower their emissions. Thus, if cooperation among these three major actors becomes annoyed, the agreement can be at risk of being undermined. Similarly, the US and its stability in terms of commitments is another challenge that faces the Paris Agreement. As the US government has withdrawn from Kyoto Protocol after its signature, the Supreme Court of the US has made a decision in regard to implementation of Obama's Clean Power Plan. This creates uncertainty in regard to the US and its ability to meet the intended IDC to its best quality. More recent evidence is the Trump's intention to withdraw from Paris Agreement. This can be a disaster in future of the earth as US is top three in emission of carbon dioxide and other greenhouse gases. This requires further assistance from UN or other powerful parties in terms of retaining the US in the agreement and its movement towards the reduction of emissions, meeting its intended IDC and to act as an adequate state in this manner.

Additionally, the fund of $\$ 100$ billion USD/year for financing endeavors against climate change by 2020 , alongside "loss and damage" was considered as a stimuli for nations being developed for participating and involving in the Paris Agreement. However, based on reports, this fund has been merely over \$10 billion USD along with a vivid statement that "involve or provide basis for liability or compensation" has not been the case. These issues challenge the relevance of the Paris Agreement. Likely, a study conducted by Heritage Foundation Project in 2017 stated negative long-term results in terms of financial means for US citizens as well as its energy costs (such as electricity). Loss of jobs, especially in manufacturing industries by 2035, leads to understanding that Americans can have costs regarding consumer goods, paper products, which in turn lowers the income of households

Pledge and review can turn effective and successful if costs of failure for meeting goals of pledges to be higher than 'business as usual' benefits. Reputation with its 'soft power' is a predictor of behaviors and can aid the calculation of various factors influencing this process. Public engagement is a determinant of reputation on a substantial basis. Sensitivity towards commitment can vary from state and institutions in terms of its degree. This means that some parties are more prone to meet and fulfill their commitments when under the pressure of public, when compared to others. In the light of the above and with hopes of a better future for climate and actions against climate change, the 20th century initiated the governments to not be able to use sovereignty claims against abuses of human rights. Similarly, one can hope that 21 st century will be relatively the same in terms of greenhouse gas emissions. The legacy of Paris Agreement is not merely dependent on legal bindings and the structure of its legal matters established in 2015, but collective and sustainable actions conducted by all people from all nations is of necessity for political behaviors as well as economic states. This affects all aspects of life as climate impacts all humans and their well-being. Hence, it can be deduced that the action against climate change requires not just governments, but all people on a global scale to ensure the effectiveness of the Paris Agreement. 